\title{
Can dark matter see itself?
}

\author{
Steen Hannestad \\ NORDITA, Blegdamsvej 17, DK-2100 Copenhagen, Denmark
}

(October 30, 2018)

\begin{abstract}
Many independent high-resolution simulations of structure formation in cold dark matter models show that galactic halos should have singular core profiles. This is in stark contrast with observations of both low- and high-surface brightness galaxies, which indicate that the dark matter has almost constant density in the central parts of halos. Basically there are three possible avenues to a solution to the problem, which we discuss in turn. Observations of halo profiles could be more uncertain than previously thought, and higher resolution observations could reveal that spirals do have a singular core feature. The highest resolution simulations do not include a baryonic component, and it is conceivable that violent star formation processes and similar phenomena can destroy the singular dark matter core and lead to an almost constant density core profile. Finally, we discuss in more detail the intriguing possibility that the discrepancy hints at some new exotic physics of the dark matter. Warm dark matter and self-interacting dark matter are two of the most promising candidates.
\end{abstract}

\section{INTRODUCTION}

Dark matter seems to be a necessary ingredient for structure formation in the universe [1]. The standard hierarchical clustering model where the dark matter is in the form of very non-relativistic, collisionless particles has been very successful in explaining structure from galactic scales to the largest scale observable, that of the cosmic microwave background radiation (CMBR) [1,2]. In the 1980s it was realised that the flat, pure CDM model produces too much small scale structure if it is normalized on large scales [3]. Some modification of the model is needed, and several possible avenues for this exist. The most popular model initially was mixed dark matter [4], where some of the dark matter is made up of light particles with large free streaming length. The prime candidate for these light particles is a neutrino with a mass of a few eV. However, recent results from Super-Kamiokande [5] and other neutrino oscillation experiments suggest that the mass of the most massive neutrino is of the order $0.1 \mathrm{eV}$, far too small a mass to be cosmologically significant. Thus, the mixed dark matter model is currently disfavoured although perhaps not completely excluded. However, data from type Ia supernovae at high redshift suggests that the energy density in the universe is dominated by a cosmological constant [6, 7]. This also dampens small scale structure formation and can bring the CDM model into agreement with observations without the need for hot dark matter.

Recently, however, a new problem for the CDM model has surfaced, having to do with structure on galactic scales and below. Observations of spiral galaxies, both high and low surface brightness, suggest that the dark matter in spirals is in the form of a halo with almost constant central density [8] 12]. To a reasonable approximation it can be described by a universal profile of the form $[12$

$$
\rho(r) \simeq \rho_{0} \frac{r_{0}^{3}}{\left(r+r_{0}\right)\left(r^{2}+r_{0}^{2}\right)} \rightarrow_{r \rightarrow 0} \rho_{0},
$$

where the central density, $\rho_{0}$, and the core radius, $r_{0}$, depend on the specific system. However, numerical simulations of halo formation consistently show much more singular core profiles. The first large scale study of CDM halo profiles, with sufficient resolution to resolve galaxy sized halos, was that of Navarro, Frenk and White [13, who found that the simulated CDM halos also follow a universal profile

$$
\rho(r) \simeq \frac{\rho_{c} \delta_{c}}{\left(r / r_{s}\right)\left(1+r / r_{s}\right)^{2}} \rightarrow_{r \rightarrow 0} \rho_{c} \delta_{c} \frac{r_{s}}{r},
$$

known as the NFW profile. $\rho_{c} \delta_{c}$ and $r_{s}$ are again parameters that are to be fitted to individual systems. Close to the center this profile approaches $\rho \propto r^{-1}$, in strong disagreement with observations. More recent simulations with much higher resolution find an even steeper profile, approaching $\rho \propto r^{-3 / 2}[14$ [17. Furthermore, it has been claimed that these CDM simulations are now of sufficiently high resolution that the results for the central halo profile have converged to the infinite resolution limit.

In addition to this problem with the halo density profiles, observations show that a galaxy like the Milky Way contains almost an order of magnitude fewer small satellite galaxies than what is found in CDM simulations [18, 19 . 


\section{POSSIBLE SOLUTIONS}

This discrepancy between CDM simulations and observations of galaxies is so serious that it needs to be investigated more closely, because it could indicate a fundamental flaw in our understanding of structure formation and the nature of dark matter. There are basically three possible ways to remedy the discrepancy, which we shall discuss in turn.

\section{A. Observational solutions}

Until very recently, relatively little effort has been devoted to measuring reliable rotation curves in the inner parts of low surface brightness (LSB) galaxies, the reason being that the outer parts of the rotation curves are completely dominated by dark matter, and thus hold important information about the total dark matter mass in the halo. Even if there is a singular core in the halo, its integrated mass is only a small fraction of the total halo mass. In that case, high angular resolution in the central part of the galaxy is not an important issue. Therefore observations of galactic rotation curves have primarily been made using observations of the $21 \mathrm{~cm}$ neutral hydrogen hyperfine-structure line with radio telescopes. These observations have the advantage that neutral hydrogen systems extend further out in most galaxies than the visible light, meaning that the halo structure can be mapped out to very large radii. If the total halo mass is the parameter to be determined then this technique is indeed the best possible. But because of the poor angular resolution of radio observations there are indeed significant effects of beam-smearing in the measurements of the inner rotation curves. Furthermore there is usually not much neutral hydrogen present within the central kpc of a galaxy.

Recently it was pointed out by van den Bosch and Swaters 20 that taking beam smearing into account increases the uncertainty in rotation curve measurements by a significant amount. In fact it seems that some LSB galaxies are consistent with having a cusp-like feature in the central halo, whereas others are still best fitted by a constant density core. At present it is not clear how important this beam-smearing effect really is, and other very recent studies reach the opposite conclusion, that galaxy halos are best fitted by constant density cores [11.

Instead of using radio observations to probe the inner parts of rotation curves it may be more feasible to use observations of HII regions instead of neutral hydrogen. These observations can be made with optical telescopes and in principle have very high angular resolution. HII is usually also very abundant in the central parts of galaxies. One very recent study of HII rotation curves by Swaters, Madore and Trewhella 21] indicates that there could be cusps present, but on the other hand they also do not rule out the constant density cores. Clearly, better observational data from the inner parts of galaxies are needed, as well as an increased understanding of the possible uncertainties involved in these observations.

It is more difficult to imagine an observational solution to the substructure problem. If small satellite galaxies were present in galactic halos in the numbers predicted by CDM simulations they would doubtless have been detected. However, that conclusion assumes that their mass-to-light ratio is not too different from ordinary galaxies. As will be discussed in the next section, it has been proposed that small satellite galaxies are not seen, simply because they have extremely large mass-to-light ratios.

\section{B. Astrophysical Solutions}

CDM simulations with very high resolution (sufficient to resolve cores of galactic halos) at present do not include baryons 18,17. The reason is both that the computational demands for this type of simulation are very high, and that there is no really reliable way of including star formation, supernovae and similar small scale features in the baryonic component. Without such high resolution simulations it is difficult to completely exclude the possibility that a proper treatment of the baryons would cure the discrepancy between simulations and observations.

Several scenarios have in fact been proposed that try to remedy the problem by invoking baryonic features. As was mentioned in the last section, small satellite galaxies would not have been detected if they contain very few stars. Bullock, Kravtsov and Weinberg [22] have suggested that when the universe was reionised at a redshift $z \gtrsim 5$, accretion of baryons onto existing low mass dark matter halos was almost completely halted. The presence of a strong ionising radiation field stops formation of galaxies with velocity dispersion smaller than $\simeq 30 \mathrm{~km} / \mathrm{s}$, almost exactly the scale below which the discrepancy between the predicted and observed number of systems becomes apparent. If this explanation is true, then the prediction is that galactic halos contain a very large number of dark matter clumps with very few stars in them. Such clumps could possibly have disastrous effect on disk galaxies. It is well known that thin disks are unstable to heating by the frequent passage through the disk of massive objects, whereas thick disks are 
more robust. At present it is not clear how the distribution of dark matter clumps predicted by CDM would affect disk galaxies, and better simulations of this effect would be highly desirable.

It has also been proposed that tidal interactions between baryons and dark matter in the central halo can erase the cusp-like feature seen in pure CDM simulations 23. In this scenario a singular CDM halo forms at high redshift and starts accreting a baryonic disk. At some point a massive burst of star formation and subsequent supernova events occurs, initiating a galactic wind that can eventually carry off almost all gas present in the disk. If not too much star formation has occurred prior to the wind formation, the total baryonic mass carried off could be close to the total mass of the disk. This dramatically changes the gravitational potential felt by the dark matter and the subsequent violent relaxation can erase the singular core. After this event, the baryonic disk which is observed in present day spirals is slowly accreted at lower redshift.

Even though this scenario seems plausible there are a number of serious questions related to it. Firstly, preliminary results of galactic winds driven by star formation show that almost all metals can be easily expelled from the galaxy because of their high opacity [24,25]. This fits well with the fact that metals are observed in abundance in the intergalactic medium at high redshift. However, it seems impossible to expel a large fraction of the hydrogen, which makes up most of the mass 24,25]. Thus it seems unlikely that the required amount of baryons can be expelled from the galaxy by this initial burst of star formation. Secondly, the hypothesis that most of the baryons remain in diffuse gas until they are expelled is neither supported nor ruled out by present observations. In order to determine if this is indeed the case, a better understanding of star formation is needed.

Thus, the final conclusion is that a scenario where the problem is resolved by properly taking into account interactions between baryons and the dark matter cannot be ruled out at present. However, explaining the cusp problem requires some fairly strong assumptions about the behaviour of the baryonic component at high redshift.

\section{Possible particle physics solutions}

If the astrophysical or observational solutions turn out not to work, then the only option we have left is to consider solutions from particle physics. Roughly this type of solution can be divided up into two categories, those that derive from physics at the epoch of initial power spectrum formation and those which come from changing the physical properties of the dark matter particles.

The first type of solution is in a sense the simplest because it does not affect physics during structure formation, only the initial conditions. The initial condition in this regard is the power spectrum of fluctuations from inflation. In order to solve the substructure problem we need to dramatically reduce fluctuations on scales smaller than a galaxy, or

$$
\frac{k}{1 \mathrm{Mpc}^{-1}} \gtrsim 5\left(\frac{M}{10^{11} M_{\odot}}\right)^{1 / 3} .
$$

This could for instance be the case in inflationary models with broken scale invariance, as was proposed by Kamionkowski and Liddle [26]. These authors showed that the substructure problem could indeed be solved by this method. However, the cusp problem is more serious. It has been shown in N-body CDM simulations, with an artificial power spectrum cut-off introduced, that dark matter halos also have singular cores [17]. The reason for this is simply that in any cold, collisionless collapse there is a large amount of material with very low entropy (temperature). This material eventually ends up in the centers of gravitational wells, forming the singular cores observed in simulations. Thus, the conclusion is that introducing a power spectrum cut-off does not remedy the problem, it is a generic feature in any collisionless CDM structure formation scenario, regardless of the initial conditions.

This leads us to consider the other possible solution which has to do with the nature of the dark matter itself. A very interesting possibility was proposed by Spergel and Steinhardt [27] (see also 28 34]), namely that the cold dark matter could possess relatively strong self-interactions. If there are such self-interactions they have significant effect on halo formation. For example substructure clumps will be evaporated by interactions with the smooth dark matter halo background, and could decrease the number of substructure halos to the observed level.

The maximum entropy state of any self-gravitating system is the singular isothermal sphere, regardless of possible self-interactions. However, if the interaction cross-section has the right magnitude, collapse to a singular halo could be prevented for a sufficiently long time that present day halos would not show singular cores. It has been shown in numerical simulations 353 37 that a cross section higher than

$$
\frac{\sigma}{m} \gtrsim 10^{-23}-10^{-22} \mathrm{~cm}^{2} / \mathrm{GeV}
$$

leads to quick core-collapse and formation of a singular core. On the other hand, cross sections smaller than 


$$
\frac{\sigma}{m} \lesssim 10^{-25} \mathrm{~cm}^{2} / \mathrm{GeV}
$$

will not have an observable effect on structure formation. If the cross section is fine-tuned to a value

$$
\frac{\sigma}{m} \gtrsim 10^{-24}-10^{-23} \mathrm{~cm}^{2} / \mathrm{GeV}
$$

the initial expansion of the halo core prior to collapse lasts more than a Hubble time, so that present day halos could fit observations (see A. Burkert in this volume for a more thorough discussion of this model). At present it is not entirely clear whether the scattering cross-sections needed to explain the substructure problem are the the same as are needed to explain the cusp problem. If this turns out not to be the case, then self-interacting cold dark matter is certainly disfavoured.

Another possibility which has received a lot of attention recently is that dark matter is not completely cold, but has some thermal motion in the early universe [38 42]. If the dark matter has thermal motion, any fluctuations are erased on scales smaller than the free-streaming length [1]

$$
k_{\text {streaming }} \simeq 1 \mathrm{Mpc}^{-1}(m / 1 \mathrm{keV})^{3 / 4},
$$

for particles which are in a relativistically decoupled thermal distribution. In the old hot dark matter model of structure formation, where the dark matter was in the form of neutrinos with a mass of $\sim 30 \mathrm{eV}$, the free-streaming length would be of order $k_{\text {streaming }} \simeq 0.05 \mathrm{Mpc}^{-1}$, roughly the size of a large cluster. This is clearly too large to fit large scale observations, but if the mass of the dark matter particle is $\sim 1 \mathrm{keV}$, the free-streaming length is of the order $k_{\text {streaming }} \simeq 1-2 \mathrm{Mpc}^{-1}$, just what is needed to explain the substructure problem. At first sight this "warm" dark matter (WDM) model [38 45] would seem to suffer from the same problem as those with a power spectrum cut-off, namely that low entropy material clusters in the central parts of halos and produce singular cores. However, there is one vital difference between this model and CDM with a power spectrum cut-off. The warm dark matter has finite temperature, which implies that the phase-space distribution occupies a finite volume in momentum space. On the other hand, the CDM distribution is by definition a $\delta$-function in momentum space. A useful quantity describing this is the average phase space density [38.,39]

$$
Q \equiv \frac{\rho}{\left\langle v^{2}\right\rangle^{3 / 2}}\left\{\begin{array}{cc}
<\infty & \text { for WDM } \\
\equiv \infty & \text { for } \mathrm{CDM}
\end{array} .\right.
$$

In an adiabatic collapse to a lowered isothermal sphere, the central halo density can be crudely estimated as 38, 39]

$$
\rho_{0} \simeq Q(3 \sigma)^{3 / 2}\left\{\begin{array}{ll}
<\infty & \text { for } \mathrm{WDM} \\
\equiv \infty & \text { for } \mathrm{CDM}
\end{array} .\right.
$$

Thus, WDM halos are expected to have non-singular cores. However, the question is whether the same WDM particle mass can solve both the substructure problem and the cusp problem. From studies of galactic substructure, as well as the Lyman- $\alpha$ forest [4] it has been concluded that $m_{\mathrm{WDM}} \gtrsim 750 \mathrm{eV}$ in order to fit observations.

To date no N-body simulation with sufficient resolution to tackle the WDM effect on halo cores has been performed. We are therefore for the moment forced to rely on crude estimates of the effect, such as Eq. (9). Hogan and Dalcanton 38,39] have estimated that the WDM mass should be $m_{\mathrm{WDM}} \simeq 200-300 \mathrm{eV}$ in order to produce the correct core radii for low surface brightness galaxies.

It therefore seems that the collisionless WDM model has difficulty in explaining both the substructure and the cusp problem (however, see also Ref. [46]). However, it should not be immediately dismissed because whereas the estimate of the mass needed to reproduce substructure is probably quite accurate, the estimate of the mass needed to resolve the cusp problem is at best a crude guess.

If it turns out that collisionless WDM does not work then WDM with self-interactions might [47]. Even relatively weak self-interactions will keep the WDM distribution in pressure equilibrium until $T \ll m$ in the early universe. In that case the WDM particles cannot free-stream and perturbation on small scales are not damped. However, the model does not resemble CDM either because the pressure equilibrium prevent fluctuations smaller than the Jeans mass from growing [48 50]. Since the Jeans length is almost a factor of 2 smaller than the free streaming scale 447]

$$
\begin{aligned}
k_{J} & \simeq 1.7 m_{\mathrm{keV}}^{3 / 4} \mathrm{Mpc}^{-1} \\
k_{\text {stream }} & \simeq 1.1 m_{\mathrm{keV}}^{3 / 4} \mathrm{Mpc}^{-1},
\end{aligned}
$$

we find that the mass needed to explain the substructure problem in this model is only $m \simeq 300-400 \mathrm{eV}$, which is quite close to the mass needed to explain finite cores. 


\section{DISCUSSION}

We have reviewed the nature of the apparent discrepancy between CDM simulations of galactic halos and observations of spiral galaxies. In addition we have discussed which possible solutions there are to this problem. The simplest possibility is that either observations or simulations are in error, but presently it is not at all clear whether or not this is indeed the case. If it turns out that the discrepancy persists even with better simulations and observations, the only other solution is some additional particle physics. Merely changing the initial power spectrum from inflation does not work, so the only option is that the dark matter is not standard CDM. Both self-interacting CDM and warm dark matter are intriguing possibilities, although at the moment these models have not been sufficiently investigated

to determine whether they work better than CDM. In any case, the CDM problem on small scales has stirred a lot of interest recently and is likely to do so in the future as well.

[1] J. A. Peacock, "Cosmological physics", Cambridge University Press (1999).

[2] See for instance M. Gross et al., Mon. Not. R. Astron. Soc. 301, 81 (1998).

[3] G. Efstathiou, W. J. Sutherland and S. J. Maddox, Nature 348, 705 (1990).

[4] M. Davis, F. J. Summers and D. Schlegel, Nature 359, 393 (1992).

[5] Y. Fukuda et al., Phys. Rev. Lett. 81, 1562 (1998).

[6] A. G Riess et al., Astron. J. 116, 1009 (1998).

[7] S. Perlmutter et al., Astrophys. J. 517, 565 (1999).

[8] W. J. G. de Blok and S. S. McGaugh, Mon. Not. R. Astron. Soc. 290, 533 (1997).

[9] S. S. McGaugh and W. J. G. de Blok, Astrophys. J. 499, 41 (1998).

[10] T. E. Pickering et al., Astron. J. 114, 1858 (1997).

[11] P. Salucci and A. Burkert, Astrophys. J. Lett. 537, 9 (2000).

[12] A. Borriello and P. Salucci, astro-ph/0001082.

[13] J. F. Navarro, C. S. Frenk and S. D. M. White, Astrophys. J. 462, 563 (1996).

[14] R. Flores and J. R. Primack, Astrophys. J. Lett. 427, 1 (1994).

[15] J. F. Navarro, astro-ph/9807084 (1998).

[16] J. F. Navarro and M. Steinmetz, astro-ph/9908114 (1999).

[17] B. Moore et al., Mon. Not. R. Astron. Soc. 310, 1147 (1999).

[18] B. Moore et al., Astrophys. J. Lett. 524, 19 (1999).

[19] S. Ghigna et al., astro-ph/9910166 (1999).

[20] F. C. van den Bosch and R. A. Swaters, astro-ph/0006048.

[21] R. A. Swaters, B. F. Madore and M. Trewhella, Astrophys. J. Lett. 531, 107 (2000).

[22] J. S. Bullock, A. V. Kravtsov and D. H. Weinberg, astro-ph/0002214.

[23] J. Binney, O. Gerhard and J. Silk, astro-ph/0003199.

[24] M.-M. Mac Low and A. Ferrara, Astrophys. J. 513, 142 (1999).

[25] A. D'Ercole and F. Brighenti, Mon. Not. R. Astron. Soc. 309, 941 (1999).

[26] M. Kamionkowski and A. R. Liddle, Phys. Rev. Lett. 84, 4525 (2000).

[27] D. N. Spergel and P. J. Steinhardt, Phys. Rev. Lett. 84, 3760 (2000).

[28] S. Hannestad, astro-ph/9912558

[29] A. Burkert, Astrophys. J. Lett. 534, 143 (2000).

[30] C. Firmani et al., Mon. Not. R. Astron. Soc. 315, 29 (2000).

[31] N. Yoshida et al., Astrophys. J. Lett. 535, 103 (2000).

[32] B. Moore et al., astro-ph/0002308 Astrophys. J. Lett. 535, 21 (2000).

[33] J. P. Ostriker, astro-ph/9912548

[34] J. Miralda-Escude, astro-ph/0002050

[35] B. D. Wandelt et al., astro-ph/0006344

[36] R. Davé et al., astro-ph/0006218

[37] N. Yoshida et al., astro-ph/0006134

[38] C. J. Hogan and J. J. Dalcanton, astro-ph/0002330.

[39] J. J. Dalcanton and C. J. Hogan, astro-ph/0004381

[40] J. Sommer-Larsen and A. Dolgov, astro-ph/9912166 (1999).

[41] V. K. Narayanan, D. N. Spergel and R. Davé, astro-ph/0005095.

[42] P. Colin, V. Avila-Reese and O. Valenzuela, astro-ph/0004115. 
[43] R. Schaefer and J. Silk, Astrophys. J. 332, 1 (1988).

[44] S. Colombi, S. Dodelson and L. M. Widrow, Astrophys. J. 458, 1 (1996).

[45] S. D. Burns, astro-ph/9711304.

[46] J. Madsen, astro-ph/0006074.

[47] S. Hannestad and R. J. Scherrer, Phys. Rev. D 62, 043522 (2000).

[48] E. D. Carlson, M. E. Machanek and L. J. Hall, Astrophys. J. 398, 43 (1992).

[49] M. E. Machacek, Astrophys. J. 431, 41 (1994).

[50] A. A. de Laix, R. J. Scherrer and R. K. Schaefer, Astrophys. J. 452, 495 (1995). 